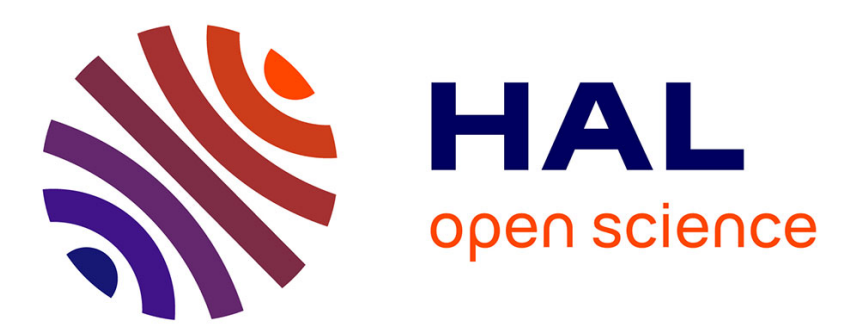

\title{
Synchronisation d'un laser à colorant en impulsions par injection d'un faisceau laser continu de grande pureté spectrale
}

\author{
F. Tréhin, G. Grynberg, B. Cagnac
}

\section{- To cite this version:}

F. Tréhin, G. Grynberg, B. Cagnac. Synchronisation d'un laser à colorant en impulsions par injection d'un faisceau laser continu de grande pureté spectrale. Revue de Physique Appliquée, 1978, 13 (6), pp.307-312. 10.1051/rphysap:01978001306030700 . jpa-00244455

HAL Id: jpa-00244455

https://hal.science/jpa-00244455

Submitted on 1 Jan 1978

HAL is a multi-disciplinary open access archive for the deposit and dissemination of scientific research documents, whether they are published or not. The documents may come from teaching and research institutions in France or abroad, or from public or private research centers.
L'archive ouverte pluridisciplinaire HAL, est destinée au dépôt et à la diffusion de documents scientifiques de niveau recherche, publiés ou non, émanant des établissements d'enseignement et de recherche français ou étrangers, des laboratoires publics ou privés. 


\title{
SYNCHRONISATION D'UN LASER À COLORANT EN IMPULSIONS PAR INJECTION D'UN FAISCEAU LASER CONTINU DE GRANDE PURETÉ SPECTRALE (*)
}

\author{
F. TRÉHIN, G. GRYNBERG et B. CAGNAC
}

Laboratoire de Spectroscopie Hertzienne de l'E. N. S., associé au C. N. R. S., Université Pierre et Marie Curie, 4, place Jussieu, 75230 Paris Cedex, France

(Reçu le 5 décembre 1977, révisé le $1^{\mathrm{er}}$ mars 1978, accepté le 17 mars 1978)

\begin{abstract}
Résumé. - Nous présentons dans cet article les performances d'un laser à colorant pompé par flash lorsque l'on injecte dans la cavité du laser à colorant un faisceau continu de grande pureté spectrale $(\Delta v<20 \mathrm{MHz})$. Nos expériences sont réalisées en utilisant la sulforhodamine $\mathrm{B}$ comme colorant, le faisceau continu provenant d'un laser à krypton (raie à $6471 \AA$ ). La répartition spectrale du faisceau laser en impulsion dépend de plusieurs paramètres dont nous étudions l'influence : tension appliquée aux flashs, puissance injectée dans la cavité, accord de la cavité. Dans les meilleures conditions de fonctionnement, nous obtenons une puissance de 100 watts pendant $200 \mathrm{~ns}$ dans une bande spectrale inférieure à $25 \mathrm{MHz}$, la distance entre le centre de cette bande et la fréquence injectée est inférieure à $15 \mathrm{MHz}$. On remarque en outre que $5 \mathrm{~mW}$ de puissance injectée suffisent pour atteindre ces meilleures performances.
\end{abstract}

Abstract. - We present the results obtained from a flashlamp pumped dye-laser when a high spectral purity C. W. beam $(\Delta v<20 \mathrm{MHz})$ is injected into the cavity of the pulsed laser. Our experiments are performed using the dye "sulforhodamine B », the C. W. beam comes from a $\mathrm{Kr}^{+}$laser (line at $6471 \AA$ ). The spectral purity of the pulsed laser depends on several parameters : energy supplied to the flashlamps, C. W. power injected in the cavity, tuning of the cavity. We study the influence of each of these parameters. In the best conditions, we obtain a power of 100 watt, in a $200 \mathrm{~ns}$ pulse, in a bandwidth less than $25 \mathrm{MHz}$ and the centre of this line is less than $15 \mathrm{MHz}$ away from the injected frequency. Furthermore $5 \mathrm{~mW}$ of the C. W. laser are enough to reach these results.

Introduction. - De nombreuses expériences de spectroscopie de haute résolution, et notamment la spectroscopie multiphotonique sans élargissement Doppler [1], [2] nécessitent des sources de longueur d'onde accordable, possédant simultanément de très petites largeurs spectrales (de l'ordre de quelques $\mathrm{MHz}$ ) et de grandes puissances (de l'ordre du kW). Jusqu'à présent, aucune source d'un emploi facile n'offre ces caractéristiques. Les lasers à colorant pompés par laser à azote suivant le schéma de Wallenstein et Hänsch [3] fournissent des puissances de l'ordre de $100 \mathrm{~kW}$ mais les largeurs spectrales sont relativement importantes, ne serait-ce qu'à cause de la très courte durée $\tau$ (quelques nanosecondes) de l'impulsion laser, ce qui limite la largeur de la transformée de Fourier à être au moins égale à $1 / \tau$. D'un autre côté, les lasers à colorant continus pompés par laser à argon peuvent donner des largeurs spectrales très petites, inférieures à $1 \mathrm{MHz}$; mais la puissance dans ces conditions ne dépasse guère

(*) Travail effectué avec le concours de la D. R. M. E. quelques centaines de $\mathrm{mW}$. Une solution, très élégante en théorie, consiste à utiliser un laser à colorant monomode pompé par flash. En effet la longue durée de l'impulsion du laser pompé par flash permet de réduire la limite liée à la transformée de Fourier à moins de $1 \mathrm{MHz}$. Gale [4] a ainsi obtenu des largeurs de raie inférieures à $10 \mathrm{MHz}$ avec des puissances supérieures à $10 \mathrm{~kW}$. Cependant il s'avère qu'en dépit de ces incomparables avantages, ce type d'appareil n'a pratiquement jamais été utilisé pour des expériences de spectroscopie de très haute résolution. La raison en est que jusqu'à maintenant il n'a pas été possible d'obtenir un fonctionnement régulier et reproductible, en particulier en ce qui concerne le caractère monomode de l'oscillation, dans des conditions normales de travail au laboratoire. Cependant depuis notre première expérience utilisant ce type de laser [5], nous n'avions pas perdu de vue les avantages potentiels que présentaient les lasers à colorant pompés par flash.

Une idée naturelle consiste à combiner les avantages du laser continu en matière de finesse de raie et du laser 
pompé par flash en matière de puissance $\left({ }^{1}\right)$. Une première solution consisterait à amplifier dans une cellule à colorant pompée par flash le faisceau issu d'un laser continu. Cependant, le gain en un passage dans une cellule à colorant pompée par flash est relativement faible ; et cette solution exige l'utilisation de nombreux étages amplificateurs en série [6]. La seconde idée consiste à injecter le laser continu dans une cavité contenant une cellule à colorant pompée par flash de façon à obliger le laser à flash à csciller sur le mode du laser continu. Divers auteurs [7], [8] ont essayé cette technique, mais leur but n'était pas d'atteindre une très haute résolution et les lasers continus utilisés avaient une largeur de raie relativement importante. Seuls, à notre connaissance, Blit et al. [9] ont essayé, indépendamment de nous, une technique comparable dans le but d'obtenir un laser puissant de très petite largeur spectrale. Nous aurons l'occasion de comparer à plusieurs reprises dans cet article leurs résultats et les nôtres.

L'expérience que nous avons réalisée est extrêmement simple dans son principe. Nous injectons dans la cavité d'un laser à colorant pompé par flash, la lumière issue d'un laser continu monomode et nous étudions en fonction de divers paramètres (puissance injectée

(1) L'utilisation couplée du laser continu et du laser pompé par azote peut également être envisagée, mais on retrouve avec la courte durée de l'impulsion du laser à azote la limite liée à la transformée de Fourier de l'impulsion. On peut cependant s'affranchir en partie de cette limite en utilisant la méthode des franges de Ramsey, c'est-à-dire en utilisant deux impulsions dúcalées dans le temps et ayant une relation de phase bien définie. Dans ces conditions la largeur ultime de la résonance est déterminée non pas par la durée de l'impulsion individuelle, mais par le temps qui sépare les deux impulsions (cf. SALOUR, M. and Cohen-Tannoudj, C., Phys. Rev. Lett. 38 (1977) 757). dans la cavité du laser à flash, tension appliquée au flash) le caractère monomode, la répartition spectrale, et la puissance dans l'impulsion laser.

Remarquons enfin que l'intérêt essentiel du flash est en l'occurence de fournir une impulsion longue. Une autre possibilité serait de pomper le laser à colorant par un laser à xénon [10] ou par un laser à YAG. Pinard et Liberman [11] utilisent une technique où le même laser à colorant est pompé à la fois en continu par un laser à argon ionisé et en impulsions par un laser à Nd-YAG doublé en fréquence ; ils ont ainsi obtenu des puissances importantes et de petites largeurs de raie. Leur technique présente des possibilités à beaucoup d'égards semblables à la technique d'injection décrite dans cet article.

1. Le montage expérimental. - Le montage expérimental est représenté sur la figure 1 . Le laser continu utilisé pour ces premiers tests est un laser à krypton ionisé de Coherent Radiation. En fonctionnement monomode il fournit sur la raie $6471 \AA$ une puissance maximum de l'ordre de $200 \mathrm{~mW}$. En analysant avec un Fabry-Pérot la lumière issue du laser, nous pouvons donner $20 \mathrm{MHz}$ coinme limite supérieure à la largeur spectrale de la lumière issue de ce laser.

Entre le laser continu et la cavité du laser à flash, nous interposons :

- un polariseur $P$ suivi d'une lame quart d'onde afin d'éviter le couplage de la cavité du laser continu avec celle du laser à flash,

- un filtre gris de manière à pouvoir faire varier la puissance injectée dans le laser à flash,

- une lentille afin d'assurer que le mode du laser continu introduit dans la cavité du laser à flash coïncide avec un mode de cette cavité,

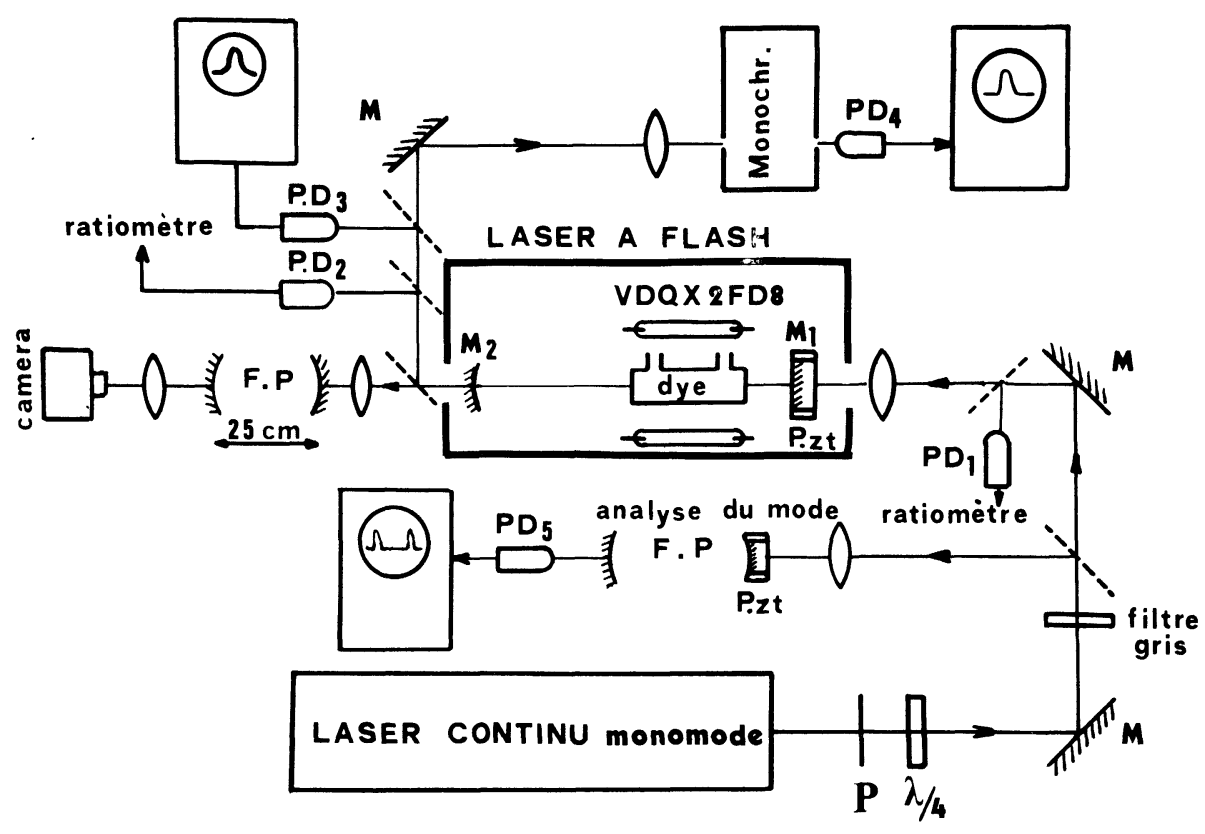

FIG. 1. - Montage expérimental.

[Experimental set-up.] 
- deux séparatrices destinées à prélever une partie de la lumière, pour mesurer la puissance issue du laser continu (photodiode PD1) et pour analyser en fréquence le mode émis (photodiode PD5).

Le faisceau lumineux issu du laser à flash est divisé à l'aide de plusieurs séparatrices, les divers faisceaux ainsi obtenus servent à :

- mesurer la puissance venant du laser continu transmise à travers la cavité (photodiode PD2),

- mesurer la puissance globale (intégrée sur toutes les longueurs d'onde) au maximum de l'impulsion laser (photodiode rapide PD3),

- analyser en fonction de la longueur d'onde l'impulsion laser en utilisant le monochromateur Jobin-Yvon HRS2 suivi de la photodiode PD4,

- analyser plus finement la répartition spectrale en utilisant un Fabry-Pérot confocal de $25 \mathrm{~cm}$ de long.

Le laser à flash lui-même comprend une cavité cylindrique bielliptique qui concentre sur le tube à colorant la lumière issue de deux flashs linéaires (quartz et silice type VDQX2FD8) refroidis par un courant d'eau.

La décharge de chacun des flashs est alimentée par une capacité de $0,1 \mu \mathrm{F}$ chargée sous une tension de 10 à $20 \mathrm{kV}$. Le taux de répétition peut atteindre 10 coups par seconde. L'alimentation électrique permet de préioniser les flashs, ce qui assure un meilleur déclenchement. Le colorant utilisé dans ces expériences est la sulforhodamine $\mathbf{B}$, dissoute dans l'eau additionnée de $3 \%$ d'ammonyx LO ; sa concentration est de $10^{-4}$ mole/litre. La circulation du colorant se fait à travers un amortisseur de vibrations à coussin d'air, dont le rôle est important pour obtenir l'accord de la cavité Pérot-Fabry du laser.

Les deux miroirs $\mathbf{M}_{1}$ et $\mathbf{M}_{2}$ de cette cavité PérotFabry ont un coefficient de réflexion égal à $R=0,94$. Le miroir $M_{1}$ est monté sur une céramique piézoélectrique, qui permet d'accorder électriquement la cavité.

En effet ce dispositif ne peut fonctionner que dans la mesure où la cavité du laser à flash est accordée sur la longueur d'onde du laser continu. Il serait possible d'asservir la cavité du laser à flash sur cette longueur d'onde, selon le dispositif utilisé au laboratoire dans d'autres expériences [12]. Mais il était plus simple de moduler alternativement la longueur du laser en envoyant une tension électrique en dent de scie sur la céramique qui porte le miroir $\mathbf{M}_{1}$, et de déclencher les flashs à l'instant où la cavité passe par l'accord.

On contrôle l'accord de la cavité à l'aide de l'intensité lumineuse transmise : les signaux des photodiodes $\mathrm{PD} 2$ et $\mathrm{PD} 1$ sont transmis à un ratiomètre qui effectue leur rapport PD2/PD1. Le signal obtenu, observé à l'oscillographe, est une cour be d'Airy analogue à celle qui est schématisée sur la figure 2. Un comparateur permet d'envoyer un signal de commande sur un générateur d'impulsions quand le signal du ratiomètre atteint la mi-hauteur du pic d'Airy (point 0 ). Ce générateur transmet l'impulsion aux flashs avec un retard variable, ce qui permet de déclencher les flashs pour différents réglages de la cavité du laser, allant de l'accord parfait (position 1) à un désaccord total (positions 3, 4, 5).

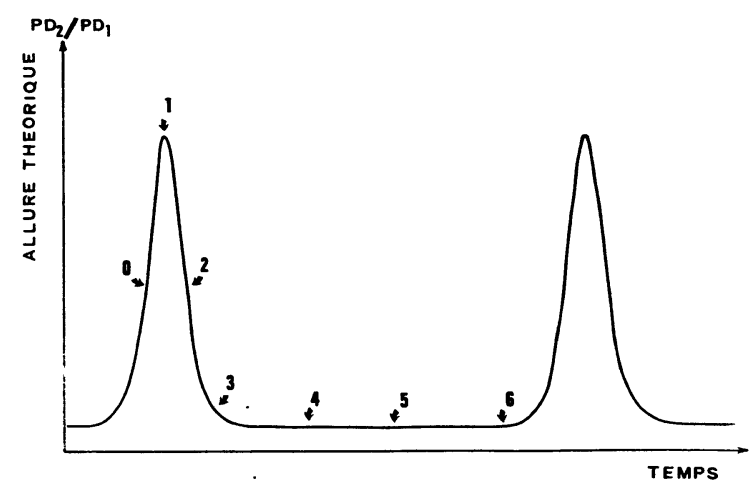

FIG. 2. - Signal théorique fourni par le ratiomètre utilisé pour le déclenchement des flashs.

[Signal coming from the ratiometer and used for the triggering of the flash-tubes.]

2. Déroulement des expériences. - Avant de commencer les expériences d'injection proprement dites, nous avons testé le fonctionnement du laser à flash seul. La durée de l'impulsion laser est d'environ $0,3 \mu \mathrm{s}$. Avec la rhodamine $6 \mathrm{G}$, la puissance lumineuse délivrée au maximum de l'impulsion atteint plusieurs kilowatts. Mais avec la sulforhodamine B utilisée dans cette expérience (imposée par la longueur d'onde du laser continu à krypton) la puissance lumineuse obtenue est environ dix fois inférieure, soit plusieurs centaines de watts.

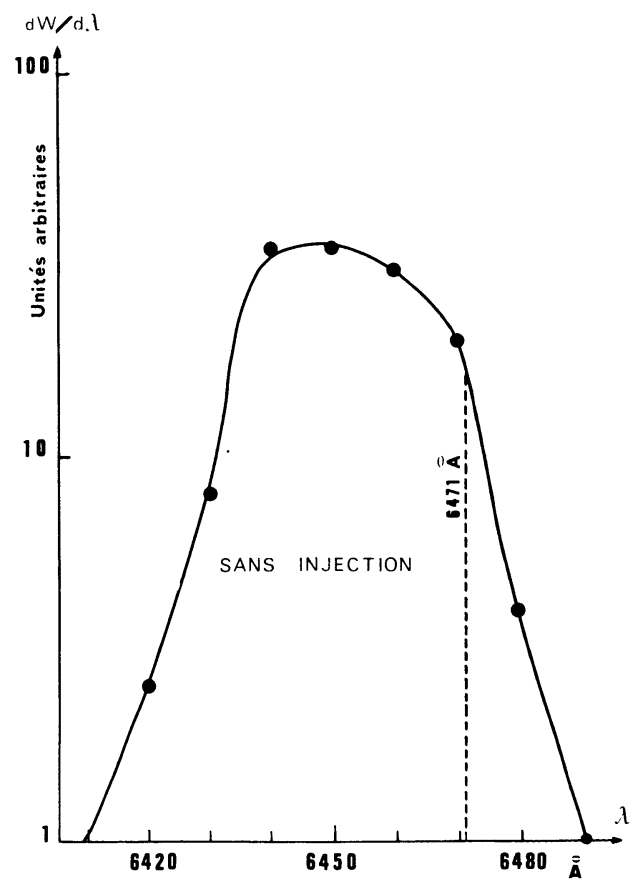

FIG. 3. - Spectre émis par le laser à flash sans injection (avec la sulforhodamine $B$ ).

[Spectrum emitted by the flash pumped dye-laser without injection (the dye is the sulforhodamine B).] 
La cavité du laser à flash ne comporte aucun élé. ment sélectif en longueur d'onde ; et cette puissance lumineuse est répartie sur un large intervalle spectral, dont la figure 3 donne un exemple : la figure 3 représente en unités arbitraires, sur une échelle logarithmique, le signal de la photodiode PD4 en fonction de la longueur d'onde sélectionnée par le monochromateur (la largeur des fentes correspondait à un intervalle spectral de $2 \AA$ ). Cette courbe a été tracée avec une valeur de la haute tension de $17 \mathrm{kV}$, voisine du seuil. Avec des tensions plus élevées la courbe tend à s'élargir mais elle reste centrée à $6450 \AA$; c'est-à-dire que l'émission normale de la sulforhodamine $B$ ne favorise pas spécialement la longueur d'onde de $6471 \AA$ A choisie pour l'injection.

En présence d'injection à la longueur d'onde de $6471 \AA$, la courbe de répartition spectrale change profondément. Dans certains cas, il peut arriver que la totalité de la puissance émise soit concentrée à la longueur d'onde imposée, c'est-à-dire qu'il se produit un accrochage total. Mais dans d'autres cas une fraction seulement de la puissance émise se trouve à la longueur d'onde injectée, tandis que l'autre fraction reproduit le spectre large de la figure 3 ; l'accrochage est seulement partiel.

Ces phénomènes sont illustrés par les photographies de la figure 4 ; elles ont été effectuées avec la caméra indiquée sur la figure 1, qui enregistre les anneaux d'une cavité Fabry-Pérot confocale de $25 \mathrm{~cm}$ de long. La photographie $4 a$ sert de référence : elle montre les anneaux formés par le laser continu seul au cours d'une pose de longue durée. Les trois autres photographies $4 b, 4 c$ et $4 d$ sont obtenues avec des temps de pose très courts coïncidant avec une impulsion du laser à flash, dans des conditions telles que la lumière du laser continu ne peut pas impressionner la plaque photographique. La photographie $4 b$ montre un cas d'accrochage total (la haute tension de $17 \mathrm{kV}$ est voisine du seuil) ; la photographie $4 c$ montre un cas d'accrochage partiel (la haute tension vaut $20 \mathrm{kV}$ ) ; la photographie $4 d$ a été faite en l'absence d'injection pour comparaison.

Nous avons fait une étude systématique des conditions d'accrochage et de la puissance émise en fonction des paramètres qui déterminent l'impulsion du laser à flash (haute tension, instant de déclenchement, puissance injectée). Faisant varier les conditions de fonctionnement du laser à flash, nous avons effectué les trois contrôles suivants :

1. - un contrôle visuel des anneaux formés à la sortie du Fabry-Pérot de $25 \mathrm{~cm}$ (sur un écran remplaçant la caméra de la figure 1) nous permet de savoir si un accrochage partiel ou total a eu lieu,

2. - le signal de la photodiode rapide PD3 nous permet, grâce à un étalonnage préalable, de mesurer la puissance lumineuse émise au maximum de l'impulsion laser, sur l'ensemble du spectre de longueur d'onde, a)

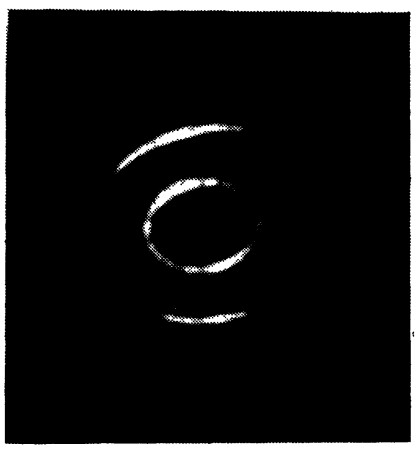

b)

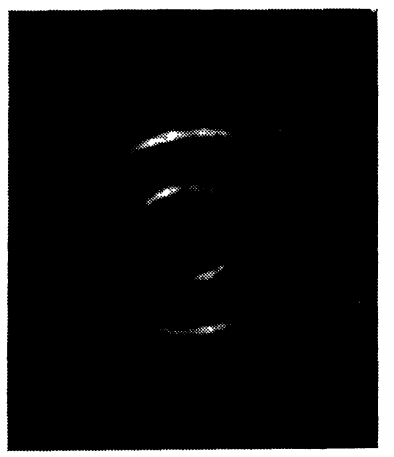

c)

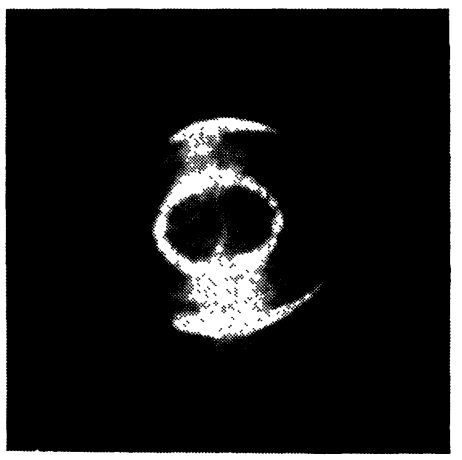

d)

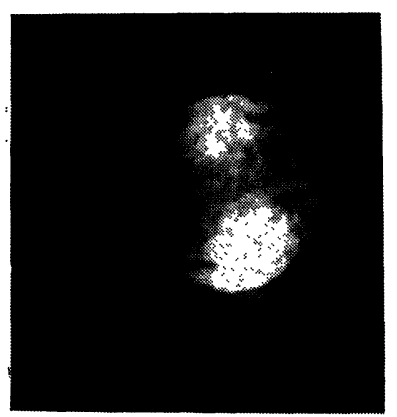

Fig. 4. - Analyse spectrale avec un interféromètre de Pérot et Fabry de la lumière émise par le laser : $a$ ) référence : laser continu seul ; b) laser à flash, cas de synchronisation totale ; c) laser à flash, cas de synchronisation partielle ; $d$ ) laser à flash sans injection.

[Spectral analysis with a Fabry-Perot interferometer of the light emitted by the laser : $a$ ) reference : $\mathrm{CW}$ laser alone; $b$ ) flash pumped laser, total synchronisation; $c$ ) flash pumped laser, partial synchronisation; d) flash pumped laser, without injection.] 
3. - le monochromateur est centré sur la fréquence d'injection $6471 \AA$; et le signal de la photodiode rapide PD4 placée derrière le monochromateur fournit une mesure séparée de la fraction de la puissance lumineuse qui est émise à la longueur d'onde d'injection. En comparant les signaux des deux photodiodes PD3 et PD4 nous obtenons un contrôle quantitatif de l'accrochage partiel illustré par la photographie $4 c$. Les résultats détaillés de cette étude sont exposés dans le paragraphe suivant.

3. Conditions d'accrochage et puissance émise. Nous exposons ici les résultats des expériences effectuées selon la méthode expliquée à la fin du paragraphe précédent.

3.1 ChOIX DE L'INSTANT DE DÉCLENCHEMENT. - Le rôle du système de déclenchement des flashs est de produire l'impulsion laser à un instant où la cavité du laser est accordée à la longueur d'onde d'injection. Mais la photodiode PD2 contrôle l'accord de cette cavité dans des conditions où le milieu colorant n'est pas soumis à l'irradiation lumineuse des flashs. L'irradiation par les flashs effectue le pompage optique du milieu colorant, et on s'attend à ce qu'elle modifie simultanément son indice de réfraction, c'est-à-dire la longueur optique de la cavité laser. Pour tenir compte de cette modification de la cavité laser au moment de l'irradiation des flashs, nous avions prévu dans le système de déclenchement un retard variable entre le signal de commande (flèche zéro sur la figure 2) et l'impulsion qui déclenche les flashs : cet instant de déclenchement peut être repéré sur la figure 2 par la position des flèches numérotées de 1 à 6 .

Nous avons essayé systématiquement ces divers instants de déclenchement en maintenant constante la haute tension d'alimentation des flashs $(17 \mathrm{kV})$ et la puissance continue injectée $(5 \mathrm{~mW})$; c'est l'instant de déclenchement correspondant à la flèche 2 de la figure 2 qui fournit les meilleures conditions d'accrochage (alors que le meilleur accord de la cavité en l'absence d'irradiation par les flashs correspond à la flèche 1). Dans ces conditions, on obtient l'accrochage pour plus de neuf impulsions sur dix.

3.2 INFLUENCE DE L'INTENSITÉ LUMINEUSE DE POMPAGE. - Nous pouvons faire varier l'intensité des flashs en changeant la haute tension avec laquelle sont chargés les condensateurs qui alimentent la décharge. Nous avons représenté sur la figure 5 les variations de la puissance lumineuse obtenue au maximum de l'impulsion laser en fonction de la valeur de la haute tension entre 15 et $20 \mathrm{kV}$. Ces mesures sont effectuées avec une puissance injectée constante égale à $5 \mathrm{~mW}$ et en utilisant le retard au déclenchement déterminé précédemment; nous éliminons les impulsions où aucun accrochage ne se produit. La figure 5 comporte trois courbes : les deux premic̀res correspondent au laser synchronisé :
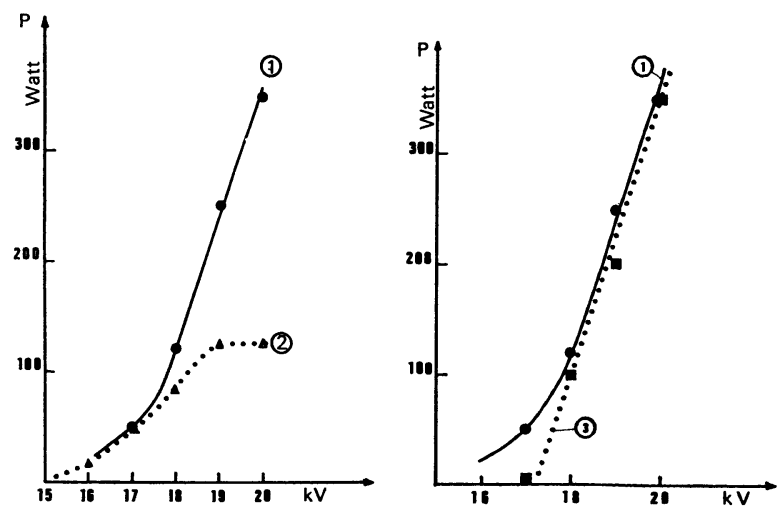

Fig. 5. - Puissance lumineuse émise par le laser à flash en fonction de la haute tension appliquée aux flashs : courbe (1) puissance totale avec injection ; courbe (2) puissance synchronisée à la longueur d'onde de l'injection $(6471 \AA)$; courbe (3) puissance totale sans injection.

[Light power emitted by the flash pumped dye laser versus the high voltage applied to the flash-tubes : curve (a) total power with injection ; curve (2) synchronized power with injection at the wavelength $6471 \AA$; curve (3) total power without injection.]

- la courbe (1) représente la puissance totale émise par le laser sur l'ensemble du spectre en longueur d'onde (photodiode PD3),

- la courbe (2) représente seulement la fraction de cette puissance qui est émise à la longueur d'onde injectée (photodiode PD4).

On constate que au voisinage du seuil, en-dessous de $17,5 \mathrm{kV}$, toute la puissance est concentrée à la longueur d'onde injectée; c'est l'accrochage total illustré par la photographie $4 b$. Au contraire, pour les valeurs supérieures de la haute tension, une large partie de la puissance est émise en dehors de cette longueur d'onde ; c'est l'accrochage partiel illustré par la photographie $4 c$. Ce résultat se comprend bien : en effet dès que l'on fournit suffisamment d'énergie à l'oscillateur il préfère les modes à haut gain plutôt que le mode injecté de gain plus faible (parce que décentré par rapport au gain du colorant, cf. figure 3). On pourra remédier à ce point en ajoutant dans la cavité laser un élément sélectif en longueur d'onde qui permettra de centrer la courbe d'émission du laser libre sur la longueur d'onde injectée.

Remarquons cependant que même pour les valeurs les plus élevées de la haute tension, un tiers au moins de la puissance émise se trouve concentrée à la longueur d'onde injectée. Les puissances indiquées sur la figure 5 ( $400 \mathrm{~W}$ de puissance totale, $130 \mathrm{~W}$ de puissance synchronisée) peuvent paraître très modérées si on les compare à celles de la réference 9. Mais il faut remarquer que Blit et al. utilisent une décharge électrique d'énergie supérieure et un autre colorant (la rhodamine 6G) dont l'efficacité, dans son domaine de longueur d'onde, est aussi bien supérieure.

Pour mieux éclairer le fonctionnement du processus de synchronisation nous comparons encore sur la figure 5 les puissances émises en présence et en l'ab- 
sence d'injection : la courbe (1) représente la puissance totale émise par le laser en présence d'injection, la courbe (3) représente la puissance totale émise par le laser en l'absence d'injection.

On voit qu'au voisinage du seuil la puissance émise en présence d'injection est bien plus importante que la puissance émise par le laser libre. Nous avons noté en outre que le fonctionnement est beaucoup plus régulier en présence d'injection. Ces résultats se comprennent bien : il est en effet normal que l'injection soit particulièrement efficace au niveau du seuil quand le nombre de photons spontanés est tout juste suffisant pour la création de l'impulsion laser. Quand on se trouve nettement au-dessus du seuil, au contraire, les puissances mesurées en présence et en l'absence d'injection deviennent comparables.

3.3 INFLUENCE DE LA PUISSANCE INJECTÉE. - Nous avons fait varier la puissance injectée entre $1 \mathrm{~mW}$ et $40 \mathrm{~mW}$. Dans l'état actuel du montage nous pouvons tirer les conclusions suivantes :

- les performances en puissance de sortie comme en pourcentage d'impulsions synchronisées ne varient pratiquement pas quand la puissance injectée est supérieure à $2 \mathrm{~mW}$,

- en dessous de $2 \mathrm{~mW}$, on note essentiellement une baisse du pourcentage d'impulsions synchronisées; mais cette baisse peut être autant due à un effet spécifique du laser qu'à une faiblesse de l'électronique du déclenchement lorsque les courants des photodiodes sont trop faibles.

On peut également comprendre ces mesures de la manière suivante: le pourcentage d'impulsions synchronisées est certainement une fonction de la puissance injectée ; cependant la partie de la courbe où la variation de ce pourcentage en fonction de la puissance injectée est importante se situe dans une zone de puissance que nous n'avons pas explorée parce que trop basse. Au-delà de $2 \mathrm{~mW}$, il y a pratiquement toujours synchronisation, ce qui veut dire que l'on a déjà atteint à cette puissance un régime de saturation. Ce résultat est extrêmement encourageant parce qu'il prouve qu'il n'est pas nécessaire d'avoir un laser continu très puissant pour obtenir les performances ultimes de l'injection dans un laser à flash.

3.4 PréCISION EN FRÉQUeNCE De LA SYNCHRONISATION. - Dans les processus de synchronisation d'oscillateurs il est courant d'observer des phénomènes d'entraînement de fréquence ; et il est important de connaître l'importance de ces phénomènes dans notre expérience. La précision limitée de nos mesures interférométriques ne nous permet pas encore d'apporter une réponse totale à cette question. Mais nous pouvons dès maintenant fixer une limite supérieure à ces phénomènes.

Nous avons vu au paragraphe 2 comment nous analysons la lumière émise dans l'impulsion laser à l'aide d'un interféromètre de Fabry-Pérot confocal de $25 \mathrm{~cm}$ de longueur (cf. photographies de la figure 4). La distance entre deux modes de ce Fabry-Pérot est de $300 \mathrm{MHz}$ et la largeur instrumentale d'un mode est de l'ordre de $20 \mathrm{MHz}$. La comparaison entre les photographies d'anneaux formés par le laser continu (Fig. $4 a$ ) et les photographies d'anneaux formés par le laser à flash (Fig. 4b) nous permet d'affirmer que :

- la largeur spectrale de l'impulsion laser est inférieure à $25 \mathrm{MHz}$,

- la fréquence centrale de l'impulsion laser coïncide avec celle du laser continu à mieux que $15 \mathrm{MHz}$.

Nous avons bon espoir que les performances réelles de l'appareil soient bien meilleures que les limites supérieures indiquées ici, et nous préparons actuellement de nouvelles expériences permettant une mesure de fréquence beaucoup plus précise. Nous prévoyons en particulier d'utiliser ces impulsions laser pour étudier une transition à deux photons sans élargissement Doppler, qui nous fournira un test très certain sur la précision en fréquence.

Remerciements. - Nous remercions F. Biraben et J. Vigué pour leurs fructueux conseils concernant cette expérience ainsi que $M$. Bassini qui a beaucoup contribué à l'amélioration des performances du laser à flash.

\section{Références}

[1] Cagnac, B., Grynberg, G. et Biraben, F., J. Physique 34 (1973) 845.

[2] Bloembergen, N. et Levenson, M. D., High-Resolution Laser Spectroscopy (Springer-Verlag) 1976, p. 315 ;

Grynberg, G. et Cagnac, B., Rep. Prog. Phys. 40 (1977) 791.

[3] Wallenstein, R. et Hansch, T. W., Opt. Commun. 14 (1975) 353.

[4] Gale, G. M., Opt. Commun. 7 (1973) 86.

[5] Biraben, F., Cagnac, B. et Grynberg, G., Phys. Rev. Lett. 32 (1974) 643.
[6] Flament, P. et Meyer, Y. H., Appl. Phys. Lett. 19 (1971) 491.

[7] Turner, J. J., Moses, E. I. et Tang, C. L., Appl. Phys. Lett. 27 (1975) 441.

[8] Burlamacchi, P. et Salimbeni, R., Opt. Commun. 17 (1976) 6.

[9] Blit, S., Ganiel, U. et Treves, D., Appl. Phys. 12 (1977) 69. [10] Levenson, M. D. et EesLey, G. L., IEEE J. Quantum. Electron. 12 (1976) 259.

[11] Pinard, J. et Liberman, S., Opt. Commun. 20 (1977) 344.

[12] Giacobino, E., Biraben, F., Grynberg, G. et Cagnac, B., J. Physique 38 (1977) 623. 